\title{
Critiques et ambivalence du recours à l'historicité chez Heidegger
}

Entre refondation radicale et résurgence métaphysique

\section{Servanne Jollivet}

\section{OpenEdition}

\section{Journals}

Édition électronique

URL : http://journals.openedition.org/alter/452

DOI : $10.4000 /$ alter.452

ISSN : 2558-7927

Éditeur :

Association ALTER, Archives Husserl (CNRS-UMR 8547)

Édition imprimée

Date de publication : 3 décembre 2017

Pagination : 79-95

ISBN : 978-2-9550449-3-3

ISSN : 1249-8947

\section{Référence électronique}

Servanne Jollivet, « Critiques et ambivalence du recours à l'historicité chez Heidegger », Alter [En ligne], 25 | 2017, mis en ligne le 01 décembre 2018, consulté le 21 avril 2019. URL : http:// journals.openedition.org/alter/452 ; DOI : 10.4000/alter.452 


\section{CRITIQUES ET AMBIVALENCE DU RECOURS À L'HISTORICITÉ CHEZ HEIDEGGER. ENTRE REFONDATION RADICALE ET RESURGENCE MÉTAPHYSIQUE}

Servanne Jollivet

Dès ses tout premiers cours, l'histoire apparaît sans conteste comme le "phénomène fondamental » sur lequel Heidegger entend s'appuyer pour renouveler intégralement le projet phénoménologique. Tout l'enjeu est en effet, dès 1919, de radicaliser la refondation husserlienne en accédant au " concret et à l'unique » et de réintégrer dans son analyse ce qui était justement exclu par Husserl, à commencer par la prise en vue de cet « ici et maintenant facticiel $»^{1}$ dont le geste phénoménologique doit désormais partir. Dans le même temps la démarche n'en doit pas moins s'assurer d'un accès à cet « originaire », qui n'a rien d'évident, en explicitant en amont les présupposés et la situation herméneutique qui sous-tendent l'analyse, patient travail de déconstruction auquel Heidegger s'attellera, essentiellement pendant la période de Marbourg qui précède la parution de $\hat{E}$ tre et Temps. Parce que la phénoménologie ne peut «se placer en dehors de l'histoire comme si elle n'en était pas affectée $»^{2}$, la " science originaire » qu'il a en vue en ces années se voit ainsi très vite redéfinie comme "connaissance "historique" en un sens radical »3. "Historique », à la fois parce que le projet phénoménologique se trouve désormais étayé sur un travail herméneutique qui intègre la

\footnotetext{
${ }^{1}$ M. Heidegger, Phänomenologie der Anschauung und des Ausdrucks. Theorie der philosophischen Begriffsbildung [GA 59], Francfort-sur-le-Main, Klostermann, 1993, p. 197.

${ }^{2}$ M. Heidegger, Kasseler Vorträge/Les conférences de Cassel (1925), édition bilingue, trad. J.-C. Gens, Paris, Vrin, 2003, p. 173.

${ }^{3} \mathrm{M}$. Heidegger, Interprétations phénoménologiques d'Aristote (Tableau de la situation herméneutique) [Rapport Natorp], trad. J.-F. Courtine, Mauvezin, TER bilingue, 1992, p. 31.
} 
prise en vue de sa propre historicité, mais également parce qu'en explicitant son propre ancrage, il peut alors prétendre avoir regagné « une relation véritable et originaire avec l'histoire, élucidée à partir de notre propre situation et facticité historique $»^{4}$.

La notion même d'historicité apparaît dès les tout premiers cours consacrés à la phénoménologie de la religion en 1921. Présentée comme « un des éléments de sens les plus significatifs et fondamentaux $\gg^{5}$ pour repenser l'existence, on la trouve initialement pensée à travers une terminologie encore diltheyenne, synonyme de "vitalité » (Lebendigkeit) ou encore, comme dans le Rapport Natorp, assimilée à l'idée d'une "mobilité fondamentale " (Grundbewegtheit) ${ }^{6}$ de l'existence. De fait on constate encore, au début des années vingt, une nette ambivalence liée à cette historicisation du projet phénoménologique, ambivalence qui ne sera tranchée qu'à mesure d'une distance plus marquée avec le projet diltheyen. Comme l'attestent ses tout premiers cours, Heidegger semble en effet très souvent osciller, pris dans une terminologie qu'il s'attachera par la suite à déconstruire, qualifiant tour à tour l'expérience phénoménologique d'absolue - de part sa radicale singularité - tout en reconnaissant son intrinsèque relativité. Il semble bien que l'on ait là un paradoxe, lié à cette indécision, ou une contradiction apparente que la reconduction à la notion d'historicité (Geschichtlichkeit) va justement permettre de dépasser et de trancher. Car si l'enjeu est bien de partir de l'irréductible et «absolue » unicité du vécu historique, cela ne signifie pas que le geste phénoménologique en vienne pour autant à être relativisé. Partant de ce qui est donné à l'expérience, du vécu et de notre radicale ipséité, l'analyse ne s'y épuise pas, pas plus qu'elle n'est amenée à s'enferrer dans une forme de solipsisme ou de " "monisme" de l'existence ${ }^{7}$.

Ce que Heidegger nomme "expérience facticielle" ne se réduit pas davantage à un vécu de conscience, pensable en termes psychologiques, qui conduirait à relativiser les acquis de l'approche herméneutique et à considérer qu'elle tombe elle-même «à la merci de la contingence $»^{8}$. Ce qui est proprement visé dans l'analyse, comme il le souligne dans un passage décisif, ce n'est pas « ce dont il est effectivement fait l'expérience en un tel sens ni même ce qui est toujours

\footnotetext{
${ }^{4}$ M. Heidegger, Phänomenologie des religiösen Lebens [GA 60], Francfort-sur-le-Main, Klostermann, 1995, p. 125, nous soulignons.

${ }^{5}$ M. Heidegger, GA 60, p. 323.

${ }^{6}$ M. Heidegger, Rapport Natorp, p. 24.

${ }^{7}$ M. Heidegger, Grundprobleme der Phänomenologie [GA 58], Francfort-sur-le-Main, Klostermann, 1992, p. 199.

8 M. Heidegger, GA 58, p. 162.
} 
appréhendé sur le mode d'un vécu » ${ }^{9}$. Il s'agit bien plutôt d'élargir pour ainsi dire l'analyse transcendantale aux dimensions de l'existence, qui trouvera sa forme la plus aboutie dans l'analytique du Dasein que l'on connaît dans $\hat{E} t r e$ et Temps. S'il entend partir de la concrétude de l'existence et d'un domaine d'expérience historiquement déterminé, ce doit être en effet toujours pour accéder à son " sens d'être », explicité à partir de structures facticielles, puis « existentiales » censées rendre possible toute expérience, fût-elle unique et singulière. Une fois hissée au rang d'existential et de structure indépassable, la notion d'historicité joue à cet égard un rôle pivot, puisqu'elle va permettre pour ainsi dire $\mathrm{d}^{\prime}$ « ontologiser » ${ }^{10} \mathrm{l}^{\prime}$ histoire en coupant court à l'indécision et à toute tentation "psychologisante », encore bien présente chez Dilthey. Il faut en effet attendre la longue recension qu'il consacre en 1924 à la correspondance échangée par Dilthey et Yorck von Wartenburg, qui sera ensuite reprise dans le volume $64^{11}$, et les conférences de Cassel (1925) 12 pour que la notion soit véritablement thématisée, analyse qui sera ensuite reprise dans ses grandes lignes dans le paragraphe 72 de Être et Temps. Et c'est également le moment où, sur le sol de la déconstruction opérée en amont pendant les cours de Marbourg, Heidegger va pouvoir maintenir de front ces deux exigences en apparence antinomiques visant à assurer à sa refondation phénoménologique une systématicité à toute épreuve tout en partant de l'ancrage historique et singulier d'une existence « hic et nunc ».

Si l'introduction de la notion d'historicité (Geschichtlichkeit) sert ici incontestablement de garde-fou et permet à l'analyse d'échapper à la relativisation tout en faisant la part belle à l'histoire, elle n'est pourtant pas sans poser un certain nombre de problèmes. Il semble en effet que l'ambivalence et les difficultés dont est grevé dès l'initiale ce geste de reconduction à l'historicité fragilise $\mathrm{d}^{\prime}$ autant plus l'analyse qu'il s'avère à terme difficilement tenable. Talon d'Achille d'une approche qui se veut encore, au moins jusqu'à $\hat{E}$ tre et Temps, «fondamentale »- fût-ce sur le sol d'une déconstruction de ses présupposés

\footnotetext{
${ }^{9}$ Ibid., p. 109.

10 Tel est notamment le constat de Fulvio Tessitore qui considère qu' « en reconduisant l'historicité et la temporalité au Dasein, Heidegger serait parvenu à la plus stricte ontologisation de l'existence et de l'histoire depuis Hegel», in F. Tessitore, "Zwischen Historismus und Geschichtlichkeit », Dilthey-Jahrbuch, vol. 8, 1992-1993, p. 355.

${ }^{11}$ M. Heidegger, "Anmerkungen zum Dilthey-Yorck Briefwechsel », repris in Der Begriff der Zeit [GA 64], Francfort-sur-le-Main, Klostermann, 2004. La recension devait initialement paraître en 1925 dans la Deutsche Vierteljahresschrift für Literaturwissenschaft und Geistesgeschichte éditée par Paul Kluckhohn et Erich Rothacker, mais n'y fut finalement pas publiée.

12 M. Heidegger, Kasseler Vorträgel Les conférences de Cassel (1925), op. cit.
} 
substantialistes - la prise en vue de l'historicité ouvre en effet à une dimension que l'analytique existentiale ne peut seule prendre en charge, et que Heidegger s'attachera à déployer dès les années trente sous la forme d'une « histoire de l'être » (Seynsgeschichte). En revenant à grands traits sur son émergence et sa genèse, notre propos vise à montrer en quoi cette ambivalence demeure intrinsèque à la notion d'historicité, ce qui expliquerait également qu'une telle "transcendantalisation » de l'histoire demeure éminemment problématique, ce que nombre de commentateurs n'ont d'ailleurs manqué de souligner. A commencer par le formalisme qu'elle introduit, qui fait à la fois toute la force de cette approche - puisqu'elle permet d'intégrer l'histoire au cœur du projet sans renoncer à une position «fondamentale »- mais en pose également les limites, qui pourront par la suite justi-fier son abandon.

\section{Genèse du recours à l'historicité}

La source de ce recours à l'historicité, bien connue, est une radicalisation de l'impulsion diltheyenne qui, s'appuyant sur le levier de la critique qu'en donne Yorck von Wartenburg, va permettre à Heidegger d'offrir à son analyse la systématicité qui lui faisait encore défaut ${ }^{13}$. En refondant le geste phénoménologique sur une explicitation de son ancrage "pré-théorique », le geste heideggérien s'inscrit en effet à plein dans la lignée du projet initié par Dilthey visant à reconduire toute objectivation théorique à "l'expérience interne». Contre l'approche empruntée aux sciences de la nature qui ne peut rendre compte de l'expérience que de "l'extérieur », et de manière mécanique, factuelle et objectivante, celui-ci tentait déjà d'en repenser la cohérence "globale », et pour ainsi dire autotélique en la rapportant à "l'unité structurée de la vie dont je fais primordialement l'expérience ${ }^{14}$. À l'ordre appauvri, schématique et rigidifié du «fait», il s'agissait alors d'opposer le dynamisme irréductible de l'expérience, ce que Dilthey nommait déjà la « vitalité » (Lebendigkeit), ce en quoi il avait déjà une conscience aiguë de « la force agissante du

\footnotetext{
${ }^{13}$ D'où le fameux mot d'ordre mis en avant dans Être et Temps proposant de «cultiver l'esprit du comte Yorck afin de mieux servir l'œuvre de Dilthey", in M. Heidegger, Sein und Zeit, Tübingen, Niemeyer, 1993, p. 404.

${ }_{14}$ W. Dilthey, Y. von Wartenburg, Briefwechsel zwischen Wilhelm Dilthey und dem Grafen Paul Yorck von Wartenburg 1877 - 1897 [Briefwechsel], Halle-sur-Saale, Niemeyer, 1923, p. 61, cité in M. Heidegger, GA 64, p. 11.
} 
passé $»^{15}$. Heidegger $n^{\prime}$ entend pas moins en radicaliser le geste. Comme il le souligne dans les conférences de Cassel qu'il lui consacre en 1925, il ne serait néanmoins pas allé jusqu'au bout, ni ne serait parvenu, rivé qu'il serait à «l'expérience historique », à " interroger l'historicité elle-même [qui n'est autre que] la question relative au sens de être, à l'être de l'étant $»^{16}$.

C'était déjà là ce que Yorck von Wartenburg reprochait lui-même à Ranke: «de ne pas souligner suffisamment la différence générique entre l'ontique et l'historique ${ }^{17}$ et de demeurer prisonnier de considérations empiriques. D'où l'intérêt pour Heidegger d'en reprendre la critique pour faire jouer, pour ainsi dire, Yorck contre Dilthey et d'en radicaliser l'intuition première. À l'instar de Dilthey, Yorck reconnait en effet que « la philosophie ne peut s'abstraire de l'historicité $[. .$.$] , que philosopher sans y intégrer l'histoire est, méthodolo-$ giquement parlant, une survivance métaphysique ${ }^{18}$. Sa perspective n'en échappe pas moins à la relativisation qui menaçait la perspective diltheyenne. Opposant l'historique à l'ontique, le sens à ce qui est (visible), Yorck proposait de distinguer toute démarche empirique, " oculaire ", à savoir rivée aux faits et aux apparences sensibles, d'une connaissance plus profonde, "connaissance des sources cachées » inaccessible à l'historien qui ignore «que l'essentiel ne se voit pas à $l^{\prime} œ i l \mathrm{nu}{ }^{19}$. Et d'en appeler à refonder radicalement la philosophie de l'histoire en la reconduisant à son fondement premier, inapparent, qu'il pense dès 1886 comme "virtualité » puis, reprenant le terme hégélien, comme " historicité » (Geschichtlichkeit) ${ }^{20}$. C'est à partir de ce fond premier qu'il se proposait alors de reconduire toutes les autres catégories, métaphysiques et objectivantes, celle de l'être y compris, qui en était lui-même dérivé.

C'est cette proximité qui lie Heidegger au projet de Yorck, très nette pendant la période de Marbourg, qui va lui permettre de prendre progressivement ses distances avec la perspective, jugée encore trop empirique et historisante, de Dilthey. Partant du constat « que le

\footnotetext{
${ }^{15}$ M. Heidegger, Kasseler Vorträgel Les conférences de Cassel, op. cit., p. 151.

${ }^{16} \mathrm{Ibid} .$, p. 171. Heidegger nuancera néanmoins son propos : «J'aimerais ici présupposer selon la conception que je m'en fais que Dilthey n'a certes pas posé la question de l'être et n'avait pas non plus les moyens de le faire, mais que cette tendance en lui était pourtant bien vivante », in Prolegomena zur Geschichte des Zeitbegriffs [GA 20], Francfort-sur-le-Main, Klostermann, 1979, p. 173.

17 W. Dilthey, Y. von Wartenburg, Briefwechsel, op. cit., p. 191, cité in M. Heidegger, GA 64, p. 10.

${ }_{18} \mathrm{Ibid} .$, p. 69, cité in M. Heidegger, GA 64, p. 13.

${ }_{19}$ Ibid., p. 109, cité par M. Heidegger, in Sein und Zeit, op. cit., p. 401.

${ }^{20}$ Voir la lettre de Yorck à Dilthey du 4 juin 1895 où il reconnaît que leur visée commune est « de comprendre l'historicité » in Briefwechsel, op. cit., p. 185.
} 
concept d'historique est polysémique et que nous n'en avons pas encore saisi phénoménologiquement le sens $»^{21}$, Heidegger va ainsi $\mathrm{s}^{\prime}$ attacher à déconstruire pas à pas la notion d'histoire pour assurer un accès à un "rapport vivant ", irréductible à toute objectivation qui peut en être faite. Si cette reconduction s'opère initialement dans le sillage de Dilthey, un pas est néanmoins franchi au milieu des années vingt. Comme il le souligne dans les conférences de Cassel, il ne s'agit pas tant d'historiciser la perspective, mais bien «d'élaborer l'être de l'historicité, l'historicité et non l'historique, l'être et non l'étant, l'effectivité et non l'effectif », question qui « ne relève en rien de la recherche historique empirique $»^{22}$.

Contre la «déshistorisation" (Entgeschichtlichung) caractérisant toute démarche théorique et objectivante, il s'agit en effet de repenser l'historique (geschichtlich) en le rapportant à sa source vive, qui n'est autre que notre propre constitution ontologique. Parce que cette «constitution fondamentale du Dasein eu égard à l'être, ne peut être lue ontologiquement qu'à partir de l'historicité ", c'est donc à partir de l'être-historique que nous sommes, sans lequel il ne pourrait y avoir d'histoire, que celle-ci doit être repensée. En l'occurrence à partir de ce qu'il nommera dans Être et Temps « la détermination ontologique la plus originaire et ultime du Dasein $»^{23}$, à savoir la temporalité sur laquelle l'historicité est sise elle-même, pierre de touche à laquelle l'analyse sera ultimement reconduite. Et Heidegger d'en conclure : « telle est donc la tâche que de comprendre l'historicité en la reconduisant à l'explication phénoménologique du temps ${ }^{24}$. Comme il le montre, c'est en découvrant cet enracinement premier dans la temporalité qu'il sera alors possible de défaire le lien indéfectible qui rive traditionnellement l'historique à ce qui est (étant) - l'effectivité de ce qui a été - pour le penser en sa dimension proprement "possibilisante » ou, pour reprendre le terme de Yorck, comme pure «virtualité ».

Une fois rabattue sur la temporalité, l'historicité va ainsi pouvoir être pensée comme structure fondamentale, qui rend possible le déploiement temporel de l'existence entre naissance et mort. Telle qu'il la présente dans Être et Temps, c'est donc un mode spécifique, nous dit Heidegger, de "temporation de la temporalité », de telle sorte que c'est toujours « en fonction du sens de cette temporalité que

\footnotetext{
${ }^{21}$ M. Heidegger, GA 60, p. 35.

${ }_{22}$ M. Heidegger, Kasseler Vorträgel Les conférences de Cassel, op. cit., p. 171.

${ }^{23}$ M. Heidegger, Sein und Zeit, op. cit., p. 44.

${ }^{24}$ M. Heidegger, GA 64, p. 4.
} 
se détermine le sens fondamental de l'historique $»^{25}$. Une fois posée la thèse d'une antécédence de la temporalité sur l'historicité - nous ne sommes pas «temporels» parce que nous nous tenons «dans l'histoire », mais inversement nous n'existons et ne pouvons exister historiquement que parce que nous sommes, au fond de notre être, temporels - l'historicité peut alors être "purement "déduite" de la temporalité originaire du Dasein ${ }^{26}$ elle-même reconduite, en dernière analyse, à notre pouvoir-mourir (Sein-zum-Tode) qui en constituerait le «fondement celé $»^{27}$. En rapportant toute histoire à sa source, à travers la constitution fondamentale du Dasein fini, Heidegger semble ainsi offrir, à l'issue de $\hat{E}$ tre et Temps, un point d'arrêt définitif au mouvement de reconduction, mais également une réponse qui tranche momentanément l'ambivalence qui grevait ce qui apparaissait initialement sous les traits d'une énigme, « l'énigme ontologique de la mobilité $»^{28}$.

\section{Critiques et ambivalences du recours à l'historicité}

Comme il l'admettra lui-même cette " construction existentiale de $l^{\prime}$ historicité $»^{29}$ n'est assurément pas sans poser problème, solution qui l'amène à la toute fin de Être et Temps, à "régler d'un geste le problème de l'histoire ${ }^{30}$, réduit à une « question purement ontologique ${ }^{31}$. En appelant à considérer l'histoire de manière non plus " historique » (historico-empirique) mais existentiale, condition ontologique dernière qui serait elle-même soustraite à cette " histoire du monde »(Weltgeschichte) qu'elle rend possible, Heidegger en viendrait ainsi à reconduire l'histoire à une "possibilité dernière " (Grundmöglichkeit), sorte de fondement ou d'invariant ultime luimême «non relatif » et soustrait à toute historicisation. Comme certains commentateurs n'ont manqué de le souligner, la radicalisation de l'historisme en direction de l'historicité, ainsi hissée au rang d'existential et de structure indépassable, semble bien ce faisant déboucher ultimement sur une " ontologie anhistorique ${ }^{32}$ qui laisse

\footnotetext{
${ }^{25}$ M. Heidegger, Rapport Natorp, p. 25.

${ }^{26}$ M. Heidegger, Sein und Zeit, op. cit., p. 377.

${ }^{27}$ Ibid., p. 386.

${ }^{28}$ Ibid., p. 389.

${ }^{29} \mathrm{Ibid} .$, p. 376.

${ }^{30}$ Ibid., p. 377.

${ }^{31} \mathrm{Ibid} .$, p. 403.

32 Günter Scholtz souligne également le paradoxe qui consiste à ce qu'un tel « historisme radical débouche sur une ontologie anhistorique, ce en quoi il s'auto-contredit », in G. Scholtz, «Zum
} 
ouverte la question même des limites d'un projet qui demeure bien en 1927, quoi qu'il en dise, encore « fondamental ».

C'est notamment le reproche que lui adresse Karl Löwith d'avoir éviter les conséquences relativistes de cette historicisation en recourant à une perspective radicalement anhistorique. En témoignerait la référence, dans Être et Temps, au «fait toujours identique qu'est la mort naturelle $»^{33}$, condition absolue susceptible d'assurer à son analyse une sorte de "stabilité préhistorique» (vorgeschichtliche Standfestigkeit) ${ }^{34}$. En faisant de la mort la pierre de touche de sa théorie de l'historicité, Heidegger aurait suspendu l'histoire à une condition prétendument "naturelle», le pouvoir-mourir du Sein-zum-Tode. Assurément une telle interprétation «naturalisante » ne tient pas, celui-ci n'ayant cessé de se défendre contre toute réification de l'analyse, s'attachant lui-même durant les années vingt à déconstruire minutieusement tout présupposé substantialiste. Plus décisive semble à cet égard la position de Gadamer, amené à voir en cette théorie de l'historicité ce qu'il nomme

un historisme de second degré qui n'oppose pas seulement la relativité historique de toute connaissance à l'exigence de vérité absolue mais, en pensant son fondement à travers l'historicité du sujet connaissant, s'assure ce faisant la possibilité de ne plus considérer la relativité historique comme une limitation de cette même vérité ${ }^{35}$.

Heidegger parviendrait ainsi, thèse qui rejoint sur ce point l'interprétation de Löwith, à "vaincre la menace du relativisme historique $»^{36}$ tout en assumant pleinement les postulats de l'historisme. L'historicité, comme le souligne Gadamer, cesserait ainsi

de fonctionner comme une détermination limite de la raison et de sa prétention à connaître la vérité. Elle devient plutôt une condition positive pour la connaissance de la vérité. Par là, l'argumentation du relativisme historique perd tout fondement véritable. L'exigence d'un critère pour une vérité absolue se voit démasquée comme une idole abstraite et métaphysique et perd toute signification

\footnotetext{
Historismusstreit in der Hermeneutik", Historismus am Ende des 20. Jahrhunderts : eine internationale Diskussion, Berlin, Akademie Verlag, 1997, p. 204.

${ }^{33}$ K. Löwith, Heidegger, Denker in dürftiger Zeit, Göttingen, Vandenhoeck \& Ruprecht, 1960, p. 67.

${ }^{34}$ Ibid., p. 188.

${ }^{35}$ H.-G. Gadamer, Wahrheit und Methode, Tübingen, Mohr Siebeck, 1960, p. 500.

${ }^{36}$ H.-G. Gadamer, Gesammelte Werke IV, Tübingen, Mohr Siebeck, 1999, p. 434 : « Nous devons à Heidegger l'idée que l'herméneutique reste non seulement la méthode englobante des sciences humaines, ce qu'elle avait été depuis Schleiermacher, Boeckh et Dilthey, mais qu'elle imprègne au plus profond les possibilités de la philosophie elle-même, nous permettant de vaincre la menace du relativisme historique en procédant à une radicalisation de la question ».
} 
méthodologique. L'historicité cesse de susciter le spectre du relativisme historique ${ }^{37}$.

Loin de regretter le formalisme et le caractère encore transcendantal de l'analyse, c'est au contraire cette "validité a priori et neutre ${ }^{38}$ octroyée à l'historicité qui permettrait de « radicaliser ontologiquement» une analyse qui viserait finalement moins à «un dépassement des apories de l'historisme » qu'à " une reprise générale de la question de l'être ${ }^{39}$. Partant des mêmes présupposés, Gadamer ne fera ensuite qu'en systématiser ce geste en l'élevant au rang de " principe herméneutique ».

Tout en reconnaissant lui-même le caractère fructueux de ce recours à l'historicité, la position de Reinhart Koselleck est en cela intéressante qu'elle entend souligner le caractère problématique de ce formalisme. Plus proche en cela de l'interprétation de Karl Löwith, $c^{\prime}$ est sa réduction à la seule sphère existentiale qu'il entend pointer qui l'aurait empêché de ressaisir la dimension «collective » propre à l'histoire. Nulle surprise que ce soit cette critique qui soit au cour de la conférence qu'il donne lui-même en 1985 à l'occasion du 85e anniversaire de Gadamer, texte qui sera ensuite publié, deux ans plus tard, sous le titre "Théorie de l'histoire et herméneutique ${ }^{40}$. Visant Gadamer, Koselleck y offre une critique des plus radicales de cette réduction " existentiale » et herméneutique de l'histoire, incapable de rendre compte de la pluralité des formes et temporalités historiques qui ne peuvent être dérivées d'une conception fondamentale de la finitude et de l'historicité ni «des modalités existentielles développées autour de la notion d'homme conçu comme Dasein ${ }^{41}$. Comme il en fait état dans un texte de 1972 :

Avant $\hat{E}$ tre et Temps, il a été quasiment fait abstraction de l'histoire. L'historicité est traitée comme une catégorie de l'existence humaine,

\footnotetext{
${ }^{37}$ Idem.

${ }^{38}$ H.-G. Gadamer, Wahrheit und Methode, op. cit., p. 267 ; trad. Vérité et méthode. Les grandes lignes d'une herméneutique philosophique, Paris, Seuil, 1996, p. 284.

${ }^{39}$ H.-G. Gadamer, Wahrheit und Methode, op. cit., p. 263 ; trad. p. 279.

${ }^{40}$ R. Koselleck, H.-G. Gadamer, Hermeneutik und Historik, Heidelberg, Winter, 1987, repris in R. Koselleck, Zeitschichten: Studien zur Historik, Francfort-sur-le-Main, Suhrkamp, 2000, p. 97118 ; trad. A. Escudier, "Théorie de l'histoire et herméneutique ", in L'expérience de l'histoire, Paris, EHESS, 1997, p. 181-199.

${ }^{41}$ R. Koselleck, «Théorie de l'histoire et herméneutique », op. cit., p. 184. Voir également « Wozu noch Historie ", in Vom Sinn und Unsinn der Geschichte : Aufsätze und Vorträge aus vier Jehrzehnten, Berlin, Suhrkamp, 2010, p. 13: «La découverte même de l'historicité comme catégorie existentiale pour caractériser la finitude humaine et ce qui est en changement permanent ne fait que repousser le problème: l'historicité est également un symptôme de ce qui reste irréductible lorsque l'on pense l'histoire ».
} 
et pourtant aucune structure intersubjective ou transindividuelle n'est encore thématisée. Bien que Heidegger souligne comment on passe de la finitude du Dasein a la temporalité de l'histoire, il ne va pas plus loin. C'est la raison pour laquelle, d'un côté, le danger d'une ontologie transhistorique de l'histoire guette derrière la catégorie, pour le moins fructueuse, qu'est l'historicitét ${ }^{4}$.

S'inscrivant dans la lignée des positions historistes les plus radicales - qui amèneront Leo Strauss, Gadamer ou encore Löwith à le taxer d' " historiste radical », " historiste de second degré » ou encore $\mathrm{d}^{\prime}$ « historiste destinal ${ }^{43}$ - Heidegger en viendrait ainsi, " en s'extrayant du temps ", à absolutiser la relativité pour mieux s'en prémunir. De fait, l'analyse heideggérienne oscillerait, d'un côté, entre une perspective «solipciste » rivée au périmètre de l'existence finie et, de l'autre, une reconstruction quasi-mythologique de l'histoire repensée sous les traits de l' « oubli de l'être » (Seinsvergessenheit). Dans les deux cas, si elle permet de pousser à bout l'historisme jusqu'à un fond dernier, cette thématisation de l'historicité semble bien déboucher sur ce que Koselleck nomme une "métahistoire » (Metahistorie), fût-elle ici non spéculative. Comme il le montre, l'historicité aurait ainsi absorbé la dimension métahistorique propre à la philosophie de l'histoire, dont elle serait à la fois la résurgence et le succédané. Sitôt sorti de la perspective existentiale et une fois abandonné le garde-fou qu'est ce recours à l'historicité, il ne serait alors pas surprenant, comme il le souligne assez justement, de la voir revenir de plein fouet sous la forme d'une histoire de l'être. De son côté, Koselleck n'entend pas lui-même abandonner le recours à des catégories quasi «métahistoriques». Mais ce doit être en vue d'approfondir l'historicité, pour ainsi dire de $l^{\prime}$ « historiciser» en dégageant, non plus des existentiaux, mais des structures susceptibles de rendre compte de la pluralité des formes possibles de notre rapport à l'histoire. Tout l'enjeu est alors, comme il le souligne,

de poursuivre l'analyse existentiale de Heidegger dans une direction qu'il n'avait pas envisagée lui-même - bref, de comprendre ce qui rend possible les histoires alors que Heidegger s'était satisfait de la catégorie d'historicité. Cette categorie avait placé pour ainsi dire l'expérience de la relativité propre à l'historisme sur une durée positivement lisible, sans contribuer à la fondation transcendantale de la diversité des histoires réelles ${ }^{44}$.

\footnotetext{
42 R. Koselleck, «Über die Theoriebedürftigkeit der Geschichtswissenschaft », in Zeitschichten. Studien zur Historik, Berlin, Suhrkamp, 2003, p. 299.

${ }^{43}$ L. Strauss, Droit naturel et histoire, Paris, Flammarion, 1986, p. 37 ; H.-G. Gadamer, Wahrheit und Methode, op. cit., p. 500 ; K. Löwith, Denker in dürftiger Zeit, op. cit., p. 166.

${ }^{44}$ R. Koselleck, «Théorie de l'histoire et herméneutique », op. cit., p. 193.
} 
S'il ne manque de reconnaître le caractère fructueux de cette thématisation de l'historicité, Koselleck se propose donc d'en radicaliser le geste, élargissant à l'histoire l'analyse à laquelle Heidegger soumettait l'existence à l'histoire, ce qui l'amène à compléter la table des existentiaux, "la palette des catégories proposées ${ }^{45}$ en direction d'une anthropologie historique bien plus complexe. Le couple d'existentiaux être-jeté (Geworfenheit) / projet (Entwurf) - hissés dans Être et Temps au rang de conditions dernières - sera ainsi intégralement repensé sous les traits d'un binôme élargi couplant ce qu'il nomme "l'espace d'expérience » (Erfahrungsraum) et "l'horizon d'attente » (Erwartungshorizont). Pensées comme des «indices des changements du temps historique lui-même $»^{46}$, ce sont ces deux catégories qui, différemment combinées, lui serviront à fixer les deux axes d'un repère apte à faire ressortir différents types ou modes de temporalité, ce que l'on trouvera ensuite thématisé notamment par François Hartog à travers la notion de « régimes d'historicité » ${ }^{47}$.

\section{L'historicité comme pivot pour une sortie de l'analytique existentiale}

Répondant, à l'issue de $\hat{E}$ tre et Temps, à la question de savoir si « le Dasein peut être entendu encore plus originairement que dans la projection de sa propre existence ${ }^{48}$, la considération de $l^{\prime}$ historicité arrive finalement à point nommé pour concrétiser l'analyse, jugée par trop étroite et « unilatérale ». Destinés à compléter l'interprétation de la temporalité, pensée sur le mode d'une projection ekstatique, les paragraphes consacrés à l'historicité visent en effet à une élaboration plus concrète en l'approfondissant en direction de l'être-jeté (Geworfenheit) en deçà duquel l'analyse ne peut remonter. S'il s'agissait jusqu'ici d'atteindre un «horizon toujours plus originaire et plus universel ${ }^{49}$, d'où le dégagement de la structure éminemment temporelle de l'existence jusqu'à son «fondement caché » qu'est l'êtrevers-la-mort, l'historicité ne vient donc pas seulement répéter l'analyse mais en approfondit la portée en creusant la temporalité (ekstatique), cette "pure possibilité d'être », en deçà d'elle-même. Heideg-

\footnotetext{
45 Ibid., p. 185.

${ }^{46}$ R. Koselleck, "Champ d'expérience et horizon d'attente", Le futur passé, Contribution à la sémantique des temps historiques, Paris, Editions de l'EHESS, 1990, p. 328, note 4.

${ }^{47}$ Cf. F. Hartog, Régimes d'historicité, présentisme et expériences du temps, Paris, Seuil, 2003.

${ }^{48}$ M. Heidegger, Sein und Zeit, op. cit., p. 372.

${ }^{49}$ Ibid., p. 26.
} 
ger est ainsi contraint de quitter le plain-pied d'une analyse qui demeurait jusque là rivée au seul « être circulaire du Dasein $»^{50}$. Certes $\hat{E}$ tre et Temps se contente encore «d'indiquer le site ontologique du problème de $l^{\prime}$ historicité ${ }^{51}$, un " pas en arrière » décisif n'en est pas moins fait, laissant entrevoir l'excédance de la question sur le questionné et, finalement, les limites de l'analytique pour penser la question de l'être (Seinsfrage).

Comme le reconnaît Heidegger, "le dégagement de la constitution d'être du Dasein demeure seulement un chemin, le but [étant] l'élaboration de la question de l'être en général »52. Autrement dit, c'est seulement en outrepassant le " point d'où [elle] jaillit et celui auquel [elle] remonte $»^{53}$ - l'existence - qu'il devient possible de remonter plus en amont, mouvement permettant d'historiciser la perspective et $\mathrm{d}$ 'initier à terme le futur «tournant» qui l'amènera à reprendre la question de l'être "comme une question de part en part historique "54. Il ne s'agira plus seulement, comme il l'écrira quelques années plus tard, de "cheminer au seuil de la question mais bien de s'y mouvoir de l'intérieur ${ }^{55}$, ce qui entraînera un remaniement intégral de l'approche encore strictement existentiale déployée dans Être et Temps, ouvrant la voie à ce que l'on pourrait nommer, à la suite de Paul Ricoeur, un possible " enrichissement de l'originaire par le dérivé »56. La notion d'historicité, à laquelle était jusque là suspendue l'analyse, ne tardera elle-même à être abandonnée, $n^{\prime}$ apparaissant rétrospectivement $\mathrm{n}^{\prime}$ avoir finalement été " qu'un chemin ${ }^{57}$, un questionnement au « caractère transitoire $»^{58}$, voire - comme il le dira plus tardivement - une « fausse piste $»^{59}$.

Une fois la finitude du Dasein creusée en direction de ce qui la conditionne, mettant au jour ce que Heidegger nomme "Geschick »destin commun ou « histoire destinale »- on comprend alors que la question de l'historicité, qui constituait jusque là à la fois le point d'Archimède et le garde-fou à laquelle était suspendue l'analyse, disparaisse au début des années trente au profit du devenir historique (Geschehen) ou de la «dispensation » (Schickung), mouvement de tem-

\footnotetext{
${ }^{50}$ Ibid., p. 315.

51 Ibid., p. 377.

${ }^{5} \mathrm{Ibid} ., \mathrm{p} .436$.

${ }^{53}$ Ibid., p. 38.

${ }^{54}$ Ibid., p. 21.

55 M. Heidegger, Nietzsche I [GA 6.1], Francfort-sur-le-Main, Klostermann, 1996, p. 29.

${ }_{56}$ P. Ricoeur, Temps et Récit III, Paris, Seuil, 1985, p. 172.

${ }^{57}$ M. Heidegger, Sein und Zeit, op. cit., p. 436.

${ }_{58}$ M. Heidegger, Besinnung [GA 66], Francfort-sur-le-Main, Klostermann, 1997, p. 414.

${ }^{59}$ M. Heidegger, Seminare [GA 15], Francfort-sur-le-Main, Klostermann, 1986, p. 344.
} 
poration plus vaste qui outrepasse le seul pouvoir projectif du Dasein. Aux structures existentiales dégagées dans Être et Temps se substituent désormais ce que Heidegger nomme des tonalités (Stimmungen) ou « marques » d'époques (Prägungen). L'histoire n'est plus tant pensée comme «historicité du monde ${ }^{60}$, constitutive du Dasein, que comme histoire même de l'ouverture - «histoire de l'être » (Seynsgeschichte) - à laquelle le pouvoir de découvrement du Dasein demeure soumis. La vérité elle-même n'est plus réduite à sa seule portée existentiale, mais désormais dotée d'une portée proprement " historiale » ou « époquale». À l'issue de Être et Temps, l'analyse est ainsi tout naturellement amenée, non seulement à éclairer sa propre situation herméneutique - qu'il déterminera par la suite comme ère de la technique ou du "nihilisme inachevé »- mais à se déployer sous la forme d'une "remémoration historique " (geschichtliche Besinnung) dont la visée dernière sera précisément, en surmontant «l'histoire de l'être ${ }^{61}$, d'ouvrir à un « nouveau commencement ».

Nombre de commentateurs n'ont manqué de souligner le caractère assurément problématique d'une telle dérivation formelle de l'histoire à partir de l'historicité, dénonçant la scission entraînée entre l'histoire empirique - objet de l'historiographie - et cette filiation souterraine, pour ainsi dire inapparente comme le serait la source, celée (verborgen), dans laquelle elle puise. N'est-on pas alors tenté d'y voir une sorte de métahistoire ou de «mythistoire» telle qu' on la trouvait déjà à l'œuvre chez Burckhardt, en lequel Heidegger verra lui-même un "véritable penseur de l'histoire (Geschichtsdenker) » ${ }^{62}$ ? N'est-ce pas là encore subsumer l'historique sous un principe transcendant qui en assurerait à la fois la continuité et le sens intrinsèque, ce en quoi il ne ferait que réitérer l'ancien dualisme sous couvert de "différence ontologique »? Il s'en suivrait alors un véritable dédoublement quant à l'histoire, sorte de «fracture ${ }^{63}$, pour reprendre l'expression de Michel Haar, autorisant non seulement le clivage, mais l'absorption de l'histoire factuelle ou empirique au profit de ce que Yorck von Wartenburg nommait lui-même la « connaissance des

\footnotetext{
${ }^{60}$ Dans la mesure où ce devenir est toujours déjà « devenir de l'être-au-monde », " l'historicité du Dasein est essentiellement historicité du monde", in M. Heidegger, Sein und Zeit, op. cit., p. 388.

${ }^{61}$ M. Heidegger, Zur Sache des Denkens, Tübingen, Niemeyer, 1969, p. 44 ; trad. in Questions IV, Gallimard, Paris, 1976, p. 74 : «L'histoire de l'être est terminée pour la pensée qui entre dans l'Ereignis ".

${ }_{62}$ M. Heidegger, Grundfragen der Philosophie: Ausgewählte "Probleme» der «Logik» [GA 45], Francfort-sur-le-Main, Klostermann, 1984 p. 43.

${ }_{63}$ M. Haar, La fracture de l'histoire. Douze essais sur Heidegger, Grenoble, Jérôme Millon, 1994.
} 
sources cachées ${ }^{64}$, de cette «virtualité » ou puissance de déploiement qui constituerait la source «secrète », inapparente et d'autant plus efficiente gisant sous la surface de l'histoire empirique ou de l'histoire vécue.

Il est en effet tentant de rapprocher la distinction faite en son temps par Burckhardt opposant à l'histoire événementielle la "véritable histoire de l'esprit», et ce que Heidegger nomme de son côté $l^{\prime}$ « histoire véritable » (eigentliche Geschichte) qu'il oppose, dans les années trente, à la «non-histoire (Ungeschichte) qui renvoie au nécessaire (Zwangsläufig), au quotidien ${ }^{65}$ et qui, rivée aux faits, ne parviendrait à faire histoire. Comme il le souligne dans le cours du semestre d'été 1934, "on ne saisit souvent du passé que le nonhistorique, les soi-disants faits ${ }^{66}$, demeurant en surface sans pouvoir accéder aux véritables impulsions motrices qui sous-tendent les événements. Comme il le souligne, cela ne signifie pas que toute «considération historique » (historische Betrachtung) perde en légitimité. Car elle demeure à maints égards nécessaire, dès lors qu'elle est mise en regard de ce qu'il nomme une "ressaisie historique" (geschichtliche Besinnung) qui permet d'éclairer l'historicité plus profonde de l'ouverture, cette source première à partir de laquelle tout se configure. En approfondissant l'histoire en direction de cette ouverture première, Heidegger fait donc un pas supplémentaire, remontant de l'historicité à sa source même, dimension non époquale de toute ouverture (Eröffnung), fût-elle chaque fois différemment marquée selon les époques. Comme il le précise dans ses Contributions à la philosophie, c'est à partir de là que l'être, à savoir l'horizon de sens que nous croyions initialement de notre fait, « doit être déterminé, indépendamment de la manière dont on se représente son devenir et son évolution, indépendamment de toute considération et explication historiennes $»^{67}$. Et c $c^{\prime}$ est à partir de ce "même»(das Selbe), cette " unité de l'Un [qui] se montre tout au long de l'histoire de l'être en des structures à chaque fois différentes ${ }^{68}$ que nous pouvons penser le devenir occidental comme une seule et même histoire. Ainsi

\footnotetext{
${ }^{64}$ W. Dilthey, Y. von Wartenburg, Briefwechsel, op. cit., p. 109, cité par M. Heidegger, in Sein und Zeit, op. cit., p. 401.

${ }^{65} \mathrm{M}$. Heidegger, Logik als die Frage nach dem Wesen der Sprache [GA 38], Francfort-sur-le-Main, Klostermann, 1998, p. 99. Cette distinction était déjà mobilisée dans Être et Temps à travers l'opposition de l'historicité propre et de la quotidienneté, reléguée au rang d'historicité impropre.

${ }^{66}$ M. Heidegger, GA 38, p. 96.

${ }^{67}$ M. Heidegger, Beiträge zur Philosophie (vom Ereignis), [GA 65], Francfort-sur-le-Main, Klostermann, 1989, p. 494.

${ }_{68}$ M. Heidegger, Nietzsche II, trad. P. Klossowski, Paris, Gallimard, 1971, p. 337.
} 
repensée, l'histoire aurait donc bien un fond premier, anhistorique et indépassable, l'Ereignis constituant pour ainsi dire un point d'arrêt de l'historicité sur lequel l'histoire elle-même n'aurait pas prise, sorte $\mathrm{d}^{\prime}$ archi-structure «quasi-transcendantale » soustraite à toute genèse empirique et historicisation.

\section{Dépassement du dualisme et pensée de l’Ereignis}

Si elle ne se contente plus seulement «d'indiquer le site ontologique du problème de l'historicité " ${ }^{69}$, la pensée «destinale » ou " époquale » semble ce faisant bien à terme hériter de difficultés qui se posaient déjà à l'échelle de l'analytique existentiale, que ce soit eu égard à l'inévitable réductionnisme qui l'accompagne, au clivage qu'elle introduit au regard de l'histoire concrète, mais également au recours à l'anhistoricité d'un Ereignis sans lequel l'idée même d'une histoire de l'être semble difficilement tenable. Faut-il en effet considérer qu'en mettant au jour un tel «fond » celé - qui, en vertu même de ce cèlement, n'a précisément rien de «fondamental »- Heidegger retomberait dans ce qu'il semble par ailleurs fustiger chez ses prédécesseurs, faisant le jeu d'une résurgence métaphysique qui lui permettrait de remonter à la source, elle-même non historique et sans histoire, à l'aune de laquelle l'ensemble de l'histoire pourrait être rassemblé et recueilli ? N'est-ce pas là une façon de réintroduire un principe d'intelligibilité interne au devenir, principe lui-même anhistorial et qui vaudrait comme une sorte $\mathrm{d}^{\prime}$ «immémorial [...] hors époque : à la fois ici et maintenant, et jadis, hors chronologie $»^{70}$ comme le soulignait déjà Michel Haar? Assurément, si «ce qui s'adresse à nous en tant qu'Ereignis est lui-même sans histoire (ungeschichtlich) ou mieux hors-destination (geschicklos) ${ }^{71}$ comme le reconnaîtra Heidegger plus tardivement. Si à la primauté du commencement (Anfang) était jointe celle, temporelle et historiquement située, du tout premier début (Beginn), il serait en effet aisé d'hypostasier l'Ereignis, accomplissement originaire plein et saturé face auquel l'histoire ne serait qu'un lieu d' «errance», marquant l'écart à chaque fois creusé entre l'origine et ses manifestations empiriques que l'originarité de l'initial continuerait, fût-il « désapproprié » (enteignet) comme à l'âge du nihilisme, de traverser et de transir.

\footnotetext{
${ }^{69}$ M. Heidegger, Sein und Zeit, op. cit., p. 377.

${ }^{70}$ M. Haar, La fracture de l'histoire, op. cit., p. 20.

${ }^{71}$ M. Heidegger, Zur Sache des Denkens, op. cit., p. 44 ; trad. p. 74.
} 
Tant que le «commencement» (Anfang) est pour ainsi dire toujours à venir, il ne peut pourtant y avoir ni origine prédéterminée contenant la totalité du devenir ni, comme chez Hegel, de point de référence localisable et privilégié à partir duquel l'ensemble de l'histoire prendrait sens. En tant qu'il en constitue la source première, luimême « hors-destination », l'Ereignis reste justement impensable sans l'histoire qu'il rend possible. "L'histoire de l'être est l'être même, et rien que celui-ci $»^{72}$ de telle sorte qu'il n'y a ni téléologie ni logique intrinsèque sous-jacente, d'une époque à l'autre, comme le présupposait Hegel lorsqu'il assimilait l'histoire au déploiement de l'esprit absolu dans le temps. Plutôt que de constituer une unité homogène et continue, les époques ne formeraient elles-mêmes qu'une «libre suite ${ }^{73}$, marquée par la profonde discontinuité des «voltes» de l'être. Ne pouvant être réduite «à un devenir, pensé comme développement et processus $\gg^{74}$, $l^{\prime}$ histoire de l'être ne serait donc pas tant en concurrence avec l'histoire empirique qu'elle s'y articulerait, sorte de dynamique de fond qui constituerait l'envers, inapparent, d'une seule et même histoire.

Loin de déboucher sur une "méta-histoire», la pensée de l'Ereignis, de part son anhistoricité foncière, couperait ainsi court à tout dédoublement, tout comme l'historicité permettait déjà, "en ramenant $\mathrm{l}^{\prime}$ "ontique", aussi bien que "l'historique", à une unité plus originaire $\gg^{75}$, de trancher l'ambivalence qui grevait initialement l'analyse. De fait, s'il distingue l'histoire "historisante» de l'histoire « réelle » ou vécue, Heidegger est bien amené, sinon à dépasser, tout du moins à déplacer le dualisme ou la « fracture » perceptible dans la conception de Burckhardt lorsqu'il distinguait l'histoire empirique de son envers spirituel. Car il ne s'agit pas tant ici de dévaluer l'histoire empirique au profit d'une histoire plus profonde, hiéro- ou cryptohistoire qui en révèlerait la logique intrinsèque. Comme il le précise lui-même, " cette non-histoire n'est en rien quelque chose de négatif, étant bien plutôt à l'égard de l'histoire ce que la vallée est à la montagne $[. .$.$] elle est ce qui saute \mathrm{d}^{\prime}$ abord aux yeux ${ }^{76}$. Pour ensuite ajouter, faisant pour ainsi dire basculer la perspective, qui n'a alors

\footnotetext{
72 M. Heidegger, Nietzsche II, Francfort-sur-le-Main, Klostermann, 1997 ; trad. P. Klossowski, Paris, Gallimard, 1971, p. 398.

${ }^{73}$ M. Heidegger, «Qu'est-ce que la philosophie ? », in Questions II, Paris, Gallimard, 1968, p. 26.

${ }^{74}$ M. Heidegger, Der Satz vom Grund, Pfullingen, Neske, 1957, p. 109.

75 M. Heidegger, Sein und Zeit, op. cit., p. 403.

${ }^{76} \mathrm{M}$. Heidegger, GA 38, p. 99. En ce sens « ce que nous nommons ici la non-histoire ne doit pas, malgré l'expression négative, être dépréciée ou dévaluée moralement [...]. Quelque chose de moralement bon peut être radicalement non-historique et quelque chose d'immoral à l'inverse foncièrement historique », in ibid., p. 96.
} 
plus rien d'une approche classiquement spéculative, que ce n'est qu'en vertu d'une décision que nous pouvons nous dire historiques, "décision constamment remise en jeu entre l'histoire et la nonhistoire dans laquelle nous nous tenons $\gg^{77}$.

Loin de céder au strict dualisme opposant une histoire de surface à une histoire plus profonde, il n'y aurait donc bien qu'une histoire à laquelle la «non-histoire »- notre quotidien, l'histoire factuelle ou événementielle prise pour objet par l'historiographie - elle-même ressortit et participe. Tout comme la vallée est indissociable de la montagne ou encore l'ombre de la lumière, «toute époque a sa nonessence (Unwesen), sa non-histoire (Ungeschichte) » ${ }^{78}$, envers apparent, ontique venant dissimuler la force possibilisante qui $\mathrm{s}^{\prime} \mathrm{y}$ joue, tel un fond celé et non moins nécessaire. $C^{\prime}$ est en ce sens, comme Heidegger le souligne dans le cours qui fait pendant aux notes des Contributions à la philosophie, que «ce qui fait histoire (das Geschichtliche) est suprahistorique (über-historisch)», non pas au sens d'un "supra-temporel (Über-Zeitliche) ou de ce que nous nommons l'éternel, l'atemporel $»^{79}$. Avec ceci de paradoxal que seul ce «supra-historique » est dit "véritablement historique», par opposition à ce qu'il qualifie de «nonhistorique » (ungeschichtlich) 80 : ce qui, pris dans l'actualité du présent, ne parviendrait à faire histoire, à savoir à ouvrir à de nouvelles possibilités d'avenir. Une fois suspendu tout garde-fou, telle l'historicité qui jouait encore ce rôle dans $\hat{E}$ tre et Temps en permettant à l'analyse de se prémunir contre les conséquences relativistes d'une historicisation radicale, la réflexivité semble alors abyssale. Renvoyant ultimement, comme le fait Heidegger, à notre seul pouvoir de "décision » pour décider de ce qui est historique et ne l'est pas, c'est alors toute la question de savoir si une telle pensée de l'histoire ne demeure pas au final "résolument "vide" » qui se pose, exposée à son propre fond béant, si elle ne s'expose pas à tous les mauvais usages, et ne serait pas finalement «bien plus désarmée encore que l'analytique de l'êtrelà quand elle s'attachait à reconduire l'historicité à la temporalité originaire $»^{81}$.

\footnotetext{
77 Ibid., p. 112.

${ }^{78}$ Ibid., p. 116.

${ }^{79}$ M. Heidegger, GA 45, p. 42.

${ }^{80}$ Ce qui ne signifie pas « sans histoire » (Geschichtlos) dans la mesure où il n'y a précisément de "non-histoire que là où il y a quelque chose qui advient (wo Geschehen ist)", in M. Heidegger, GA 38, p. 95.

${ }^{81}$ J.-F. Courtine, « Historicité, philosophie et théologie de l'histoire chez Heidegger », in Après la fin de l'histoire, Paris, Vrin, 1998, p. 229.
} 